\title{
Effect of Resistance Exercise Intensity on the mRNA Expression of PGC- $1 \alpha$ Isoforms in Human Skeletal Muscle
}

Neil A. Schwarz, Sarah K. McKinley, Mike Spillane, Joshua J. Gann, Thomas L. Andre, and Darryn S. Willoughby

Exercise Biochemical Nutrition Laboratory; Department of Health, Human Performance, \& Recreation; Baylor University; Waco, TX

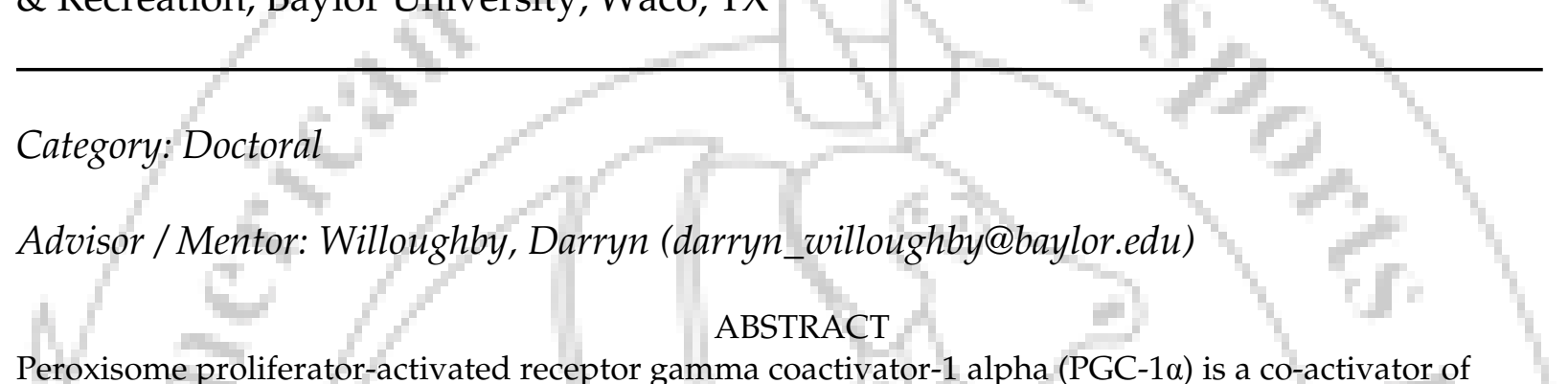

Peroxisome proliferator-activated receptor gamma coactivator-1 alpha (PGC-1 $\alpha$ ) is a co-activator of transcription demonstrated to facilitate beneficial adaptations in skeletal muscle induced by exercise, such as mitochondrial biogenesis and enhanced capillarization. Recently, novel variants of PGC-1 $\alpha$ that result from transcription initiation from an alternative upstream promoter (exon 1b) and alternative splicing of the primary transcript have been identified. The original PGC-1 $\alpha$ isoform transcribed from the canonical promoter has been designated as PGC-1 $\alpha 1$, and the novel PGC- $1 \alpha$ isoforms have been named PGC-1 2 , PGC-1 $\alpha 3$, and PGC-1 $\alpha 4$. PGC-1 $\alpha 1$, PGC-1a2 and PGC-1a3 have been shown to specifically affect energy metabolism in brown adipose tissue and skeletal muscle; whereas, PGC-1 $\alpha 4$ has been implicated to promote skeletal muscle hypertrophy. The purpose of this study was to describe the mRNA expression of PGC-1 $\alpha$ isoforms in response to two resistance exercise intensities. In a randomized, uniform-balanced, cross-over design, 10 men $[23.7 \pm 0.9$ years old (mean $\pm \mathrm{SE}$ )] performed two separate lower-body resistance exercise sessions consisting of a lower-intensity protocol (50\% of 1-RM) and a higher-intensity ( $80 \%$ of 1$\mathrm{RM}$ ) protocol with equal volume loads. Muscle samples were obtained at baseline, 45-min post-exercise (PE), 3-hr PE, 24-hr PE, and 48-hr PE from the vastus lateralis. From each muscle sample, mRNA expression of PGC-1 $\alpha 1$, PGC-1 $\alpha 2$, PGC-1 $\alpha 3$, and PGC-1 $\alpha 4$ was determined using reverse transcriptasepolymerase chain reaction (RT-PCR) and normalized to $\beta$-actin. Two-way repeated-measures ANOVA were performed $(p \leq 0.05)$ with intensity and time as main effects. Post-hoc analyses were performed using Fisher's Least Significant Difference (LSD) Test. Significant main effects existed for time $(p<.05)$, but not intensity $(p>.05)$ for all PGC-1 $\alpha$ isoforms. Additionally, no interaction effects were observed $(p>$ .05). Post-hoc analyses revealed mRNA expression of PGC-1 $1 \alpha 1$ to be decreased at 24-hr and 48-hr PE. PGC-1 $\alpha 2$ expression increased at 45-min and 3-hr PE before returning to baseline levels at 24-hr PE. PGC$1 \alpha 3$ expression increased above all other time points at 3-hr PE. Interestingly, PGC-1 $44 \mathrm{mRNA}$ expression decreased at 45-min PE before increasing to peak expression at 3-hr PE. At 48-hr PE, PGC-1 $\alpha 4$ expression returned to below baseline expression similar to expression at 45-min PE. The results of this study demonstrate PGC-1 $\alpha$ transcription to initiate exclusively from the alternative upstream promoter in response to resistance exercise. Future research is needed to describe the expression of these PGC-1 $\alpha$ isoforms at the translational level in order to help elucidate their potential role in human skeletal muscle adaptations to resistance exercise. 


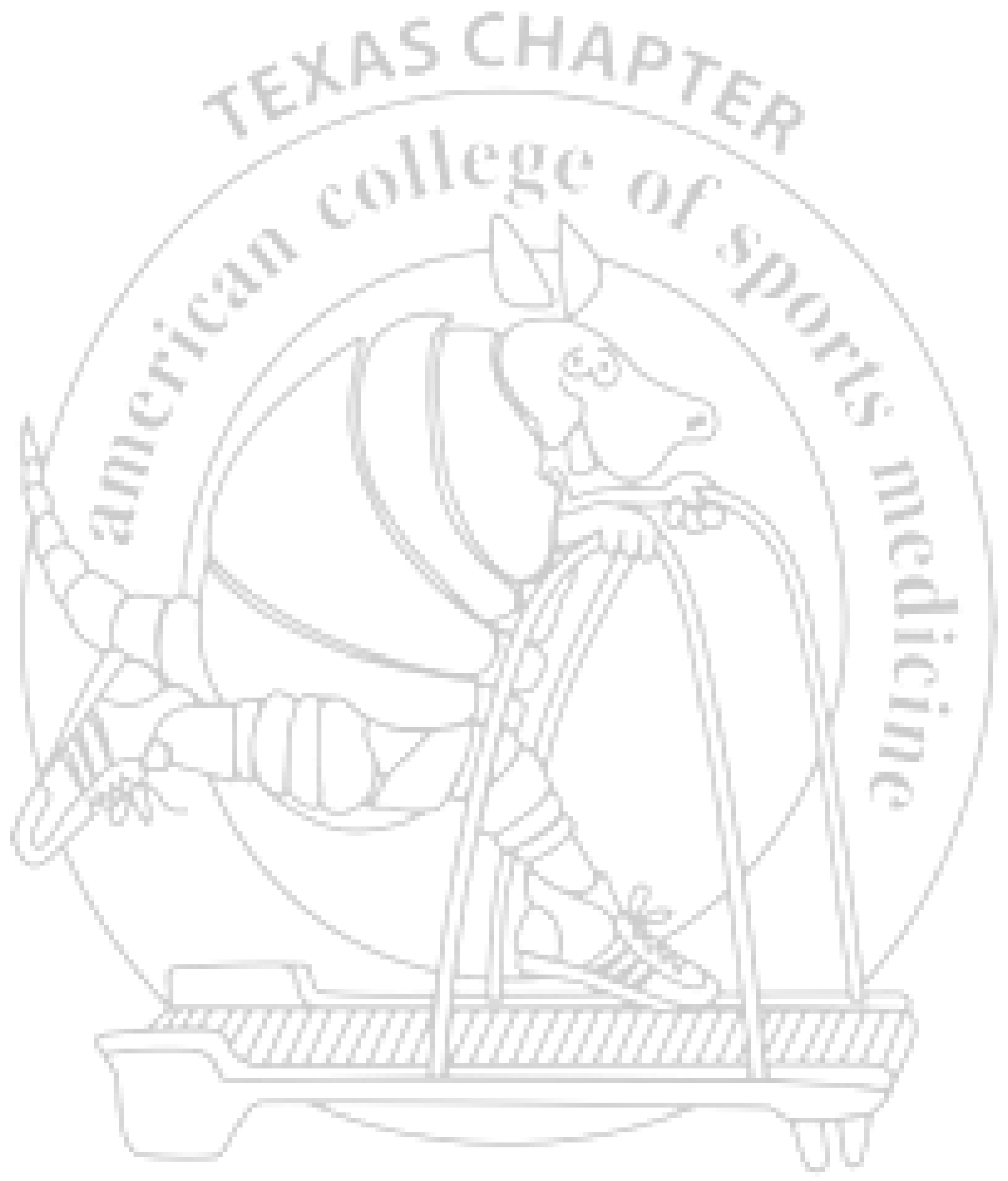

International Journal of Exercise Science

www.tacsm.org 